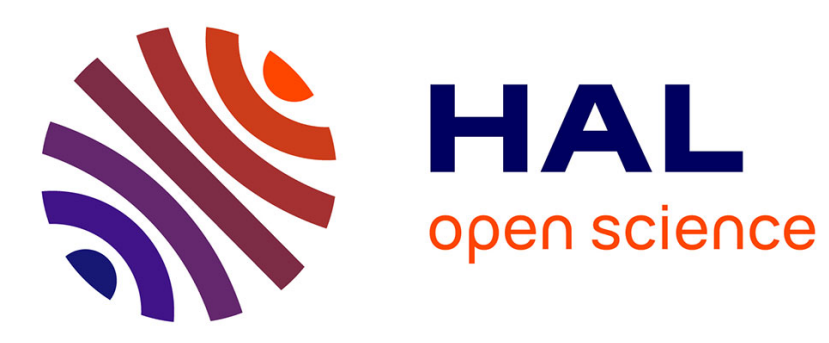

\title{
Multi-resolution Classification of Urban Areas Using Hierarchical Symmetric Markov Mesh Models
} Ihsen Hedhli, Gabriele Moser, Sebastiano B Serpico, Josiane Zerubia

\section{To cite this version:}

Ihsen Hedhli, Gabriele Moser, Sebastiano B Serpico, Josiane Zerubia. Multi-resolution Classification of Urban Areas Using Hierarchical Symmetric Markov Mesh Models. IEEE GRS/ISPRS Joint Urban Remote Sensing Event (JURSE), Mar 2017, Dubai, United Arab Emirates. hal-01415568

\author{
HAL Id: hal-01415568 \\ https://hal.inria.fr/hal-01415568
}

Submitted on 13 Dec 2016

HAL is a multi-disciplinary open access archive for the deposit and dissemination of scientific research documents, whether they are published or not. The documents may come from teaching and research institutions in France or abroad, or from public or private research centers.
L'archive ouverte pluridisciplinaire HAL, est destinée au dépôt et à la diffusion de documents scientifiques de niveau recherche, publiés ou non, émanant des établissements d'enseignement et de recherche français ou étrangers, des laboratoires publics ou privés. 


\section{Multi-resolution Classification of Urban Areas Using Hierarchical Symmetric Markov Mesh Models}

\author{
Ihsen Hedhli \\ ESPRIT, \\ PU Algorithm, Tunisia \\ Email: ihsen.hedhli@esprit.tn
}

\author{
Gabriele Moser, Sebastiano B. Serpico \\ DITEN department, \\ University of Genoa, Italy \\ Email: Gabriele.moser@unige.it
}

\author{
Josiane Zerubia \\ UCA, INRIA, \\ Sophia-Antipolis Méditerranée Center, France. \\ Email: josiane.zerubia@inria.fr
}

\begin{abstract}
In this paper we investigate a new hierarchical method for high resolution remotely sensed image classification. The proposed approach integrates an explicit hierarchical graphbased classifier, which uses a quad-tree structure to model multiscale interactions, and a symmetric Markov mesh random field to deal with pixelwise contextual information at the same scale. The choice of a quad-tree and the symmetric Markov mesh allow taking benefit from their good analytical properties (especially causality) and consequently applying time-efficient non-iterative inference algorithms.
\end{abstract}

\section{INTRODUCTION}

In this paper we address the problem of constructing statistical models of images using Hierarchical Hidden Markov modeling techniques for high resolution remotely sensed image classification of urban areas. The main difficulty is to develop a classifier that jointly utilizes the benefits of multi-band and multi-resolution input data while maintaining a good trade-off between accuracy and computation time. [1][4]. Classification techniques as examples of inverse problems solvers, can be regarded as the process that estimates hidden information (or latent variables) $\mathrm{x}$ (i.e., urban land cover class labels) from observations y (i.e., satellite data) attached to a set of nodes S. In this framework, Markov random field (MRF) models are widely used classification since they provide a convenient and consistent way of integrating contextual information into the classification scheme [5]. They represent a major methodological approach in the classification of images of urban areas because of their capability to fuse spatial-contextual and multi-scale information on the target thematic classes. Because of their generally non-causal nature, MRF models lead to iterative inference algorithms that are computationally demanding, because they require Metropolis or Gibbs sampling processes. By contrast, MRF models defined according to hierarchical structures exhibit good methodological and application-oriented properties including causality, thanks to the use of appropriate graphs [6] such as a quad-tree structure [7]. In practice, this structure sometimes yields "blocky" effects in the final classification map because of the non-adaptive topology of the quad-tree. Here, the quadtree approach is extended and a novel contextual multi-scale technique is proposed to classify multi-resolution remote sensing data that incorporates spatial contextual information and mitigates possible blocky artifacts by using a Markov mesh random field (MMRF) [8]. Unlike conventional MRFs, MMRF can be used not only to incorporate spatial information at each scale of the quad-tree but also to keep the causality of the resulting hierarchical model. However, MMRFs and their lattice models exhibit a well-known weakness, i.e., they may favor artifacts aligned with a direction departing from one corner of the image (they are "corner-dependent") [9]. Consequently, the integration of a hierarchical MRF and a spatial Markov mesh model does not necessarily exhibit an anisotropic behavior and can be affected by the use of a non-regular (non-symmetric) neighborhood. To mitigate this drawback several techniques have been recently introduced in the literature. Quadrilateral MRFs were introduced in [9], in which the non-regularity problem is avoided by using four Markov meshes related to the different corners of the lattice and enforcing them into a unique field definition; however, the model is still non-symmetric. To overcome these limitations from both mathematical and practical points of view, in [10] a new random field was established: a symmetric, corner-independent, and isotropic model that incorporates the dependency of a pixel on all its neighbors using a symmetric MMRF. The aim of the present paper is to develop a multiresolution classifier for urban areas using remote sensing data. The input images are inserted in a hierarchical structure on the basis of their spatial resolution. This approach is aimed at both exploiting multi-scale information, which is known to play a crucial role in high-resolution image analysis, and supporting contextual information at each scale.

\section{HIERARCHICAL HIDDEN MARKOV MESH MODELS}

\section{A. MRF and MMRF techniques}

Hierarchical MRFs on quad-trees rely on a causality concept captured by the factorization of the prior distribution in terms of causal transition probabilities [11]. In practice, this structure tends to generate "blocky" effects in the final classification map. To mitigate this artifact, let us consider another important class of random fields. MMRFs, also known as causal MRF or 


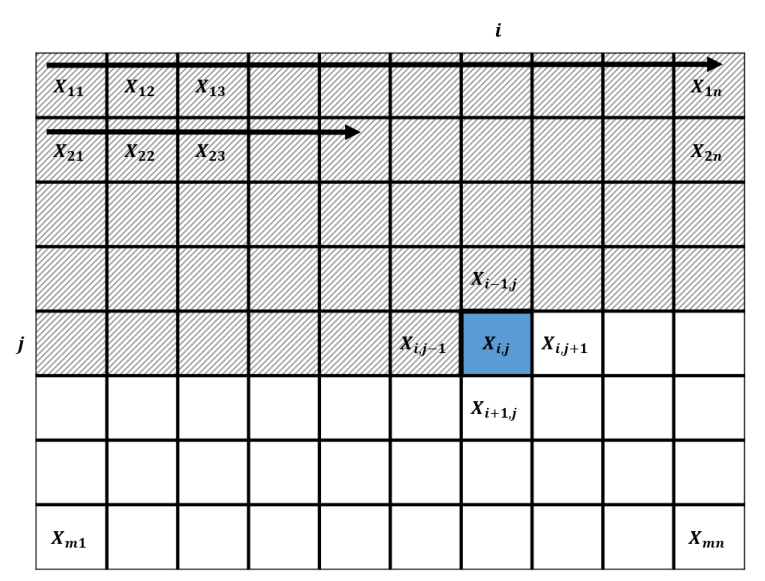

Fig. 1. Regular rectangular lattice $S$ of size $m \times n$ : the "past" of site $s_{i, j}$ is the gray area, arrow lines show raster scan.

Unilateral MRFs (UMRFs) were first introduced in [8], [12], [13]. Let us consider a finite rectangular lattice $S$ with each site $s \in S$ being associated with one or more random variables collected in a vector $x$. To build a causal process on this lattice, an order over the set of sites $S$, i.e., a well-defined characterization of the "past" of each site $s$ as a set of nodes preceding $s$, is required. As shown in (1), $X_{i, j}$ is a random variable associated with a site $s_{i, j}$ located at the coordinates $(i, j) \in[1, n] \times[1, m] . X=\left\{X_{11}, X_{12}, \ldots, X_{m n}\right\}$ represents the corresponding random field.

The probability that the random variable $X_{i, j}$ takes on the value $x_{i, j}$ is denoted by $p\left(X_{i, j}=x_{i, j}\right)$. For ease of notation this probability is abbreviated as $p\left(x_{i, j}\right)$ and the global probability is denoted by $p(X=x)=p\left(X_{11}=\right.$ $\left.x_{11}, X_{12}=x_{12}, \ldots, X_{m n}=x_{m n}\right)$ and abbreviated as $p(x)$. In this context, $X$ is said to be a MMRF if:

$$
p\left(x_{i, j} \mid p a\left(s_{i, j}\right)\right)=p\left(x_{i, j} \mid \partial\left(s_{i, j}\right)\right)
$$

where, $p a\left(s_{i, j}\right)=\left\{X_{a, b} \mid a<i\right.$ or $\left.b<j\right\}$ is considered to be the past of $X_{i, j}$ and $\partial\left(s_{i, j}\right)$ is a subset of $p a\left(s_{i, j}\right)$ that represents an unilateral neighborhood of $s_{i, j}$

For example, $X$ is a MMRF with the neighborhood size of 2 if (see Figure 1):

$$
p\left(x_{i, j} \mid p a\left(s_{i, j}\right)\right)=p\left(x_{i, j} \mid x_{i-1, j}, x_{i, j-1}\right)
$$

[12] proved that a MMRF is also a MRF while the inverse does not hold, i.e., MMRFs form a causal subclass of MRFs.

\section{B. SMMRF techniques}

Since all MMRFs are MRFs, one may intuitively expect that an MMRF with neighboring size of two is also an MRF with the neighboring size of four [Fig. 1], however this is proven to be false [12]. Indeed, the MMRF with neighboring size of two results in an abnormal unbalanced MRF behaves in "cornerdependent" way. in fact, the neighborhood dependencies in the unbalanced MRF are biased along a direction inclined of $45^{\circ}$ with respect to the horizontal axis. Consequently, the integration of a hierarchical MRF and a spatial Markov mesh does not necessarily exhibit an anisotropic behavior, which is desired in most occurrences, and can be affected by the use of a non-regular asymmetric neighborhood. Due to these disadvantages, a new hierarchical MRF based on a Symmetric Markov Mesh Random Field (SMMRF) is proposed in this paper, to overcome these limitations from both mathematical and practical points of view, and to establish a causal and symmetrical model. This can be accomplished by scanning the lattice at each level of the hierarchical model based on the visiting scheme shown in Fig. 2, as proved in [10], [14].

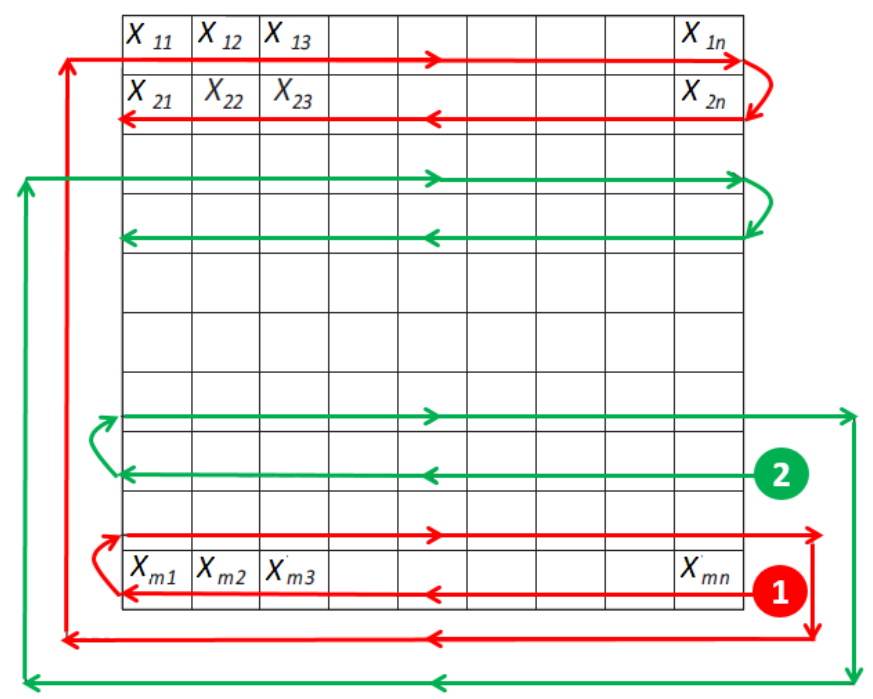

Fig. 2. Visiting the sites in SMMRF lattice to driving the model.

\section{METHODOLOGY}

\section{A. Combined Structure}

In this paper, a novel hybrid structure that combines a spatial grid using a causal SMMRF and a hierarchical MRF via a quad-tree is employed to prevent the blocky artifacts of conventional quad-tree-based methods [7] and the corner dependent artifacts of MMRFs [15]. The starting point is to define an order on the set of all nodes $S$ and a visiting scheme as in Fig 2. Then, for each scale of the quad-tree, the causal SMMRF is integrated into the hierarchical structure. Accordingly, each node $s$ at each scale level of the quadtre,e except at the root, is linked to one parent (in the upper level) and three neighbors (in the same level). For each pixel at the root level, there is no parent and only the neighbors remain. The shapes of the neighborhoods of the pixels at the top and left borders of each lattice, at each scale level of the pyramid, are obviously adapted to the image borders. The resulting dependence graph is shown in Fig. 3. 


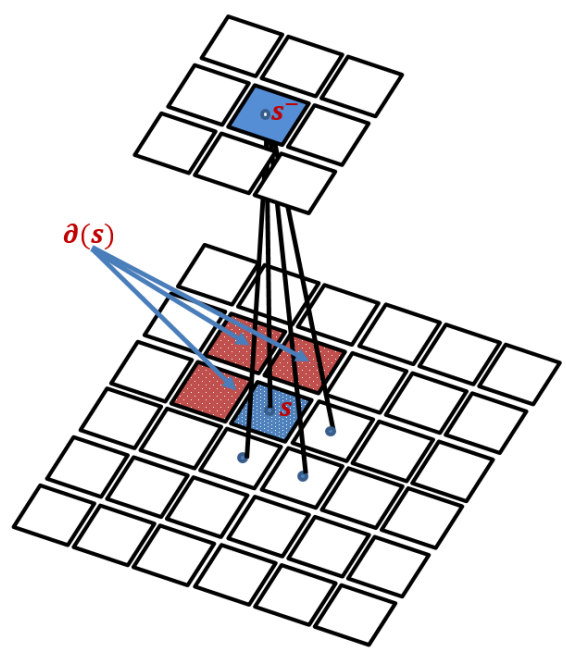

Fig. 3. Hybrid structure that combines a spatial grid using an SMMRF and a hierarchical MRF via a quad-tree.

\section{B. MPM inference}

When the causality property holds, non-iterative classification algorithms can be applied. In particular, a recursive procedure for a quad-tree is feasible for the marginal posterior mode (MPM) criterion [7], i.e.:

$$
\forall s \in S, x_{s}^{\star}=\arg \max _{x_{s} \in \Lambda} p\left(x_{s} \mid y\right),
$$

which produces the configuration that maximizes, on each site $s$, the a posteriori marginal $p\left(x_{s} \mid y\right)$ over the finite set $\Lambda$ of thematic classes in the considered urban area.

To resolve recursively the optimization problem in (3), one could use the same conditional independence assumptions as the ones developed in [16] for the case of a multi-temporal multi-resolution model. The posterior marginal $p\left(x_{s} \mid y\right)$ of the label of each node $s$ could be expressed as a function of the posterior marginal $p\left(x_{s^{-}} \mid y\right)$ of the parent node $s^{-}$in the quadtree and the posterior marginals $p\left(x_{\tilde{s}} \mid y\right)$ where $\tilde{s}$ is each one of the sites in the set $\partial(s)$ of the causal neighbors of $s$. Formally:

$$
\begin{aligned}
& p\left(x_{s} \mid y\right)= \\
& \sum_{x_{s^{-}}, x_{\partial(s)}} \frac{p\left(x_{s}, x_{s^{-}}, x_{\partial(s)} \mid y_{d(s)}\right)}{\sum_{x_{s}} p\left(x_{s}, x_{s^{-}}, x_{\partial(s)} \mid y_{d(s)}\right)} p\left(x_{s^{-}} \mid y\right) \prod_{\tilde{s} \in \partial(s)} p\left(x_{\tilde{s}} \mid y\right)
\end{aligned}
$$

The formulation in (4) allows calculating recursively the posterior marginal $p\left(x_{s} \mid y\right)$ at each node s while the probabilities $p\left(x_{s}, x_{s^{-}}, x_{\partial(s)} \mid y_{d(s)}\right)$ are produced. Thus, this simplifies to the determination of the other probabilities based on:

$$
\begin{aligned}
& p\left(x_{s}, x_{s^{-}}, x_{\partial(s)} \mid y_{d(s)}\right)= \\
& \frac{p\left(x_{s} \mid x_{s^{-}}\right) p\left(x_{s^{-}}\right)}{p\left(x_{s}\right)}\left[\prod_{\tilde{s} \in \partial(s)} \frac{p\left(x_{s} \mid x_{\tilde{s}}\right) p\left(x_{\tilde{s}}\right)}{p\left(x_{s}\right)}\right] p\left(x_{s} \mid y_{d(s)}\right)
\end{aligned}
$$

In (5), the first factor $p\left(x_{s} \mid x_{s^{-}}\right)$corresponds to the childparent transition probability; $p\left(x_{s}\right)$ is the prior probability; $p\left(x_{s} \mid x_{\tilde{s}}\right)$ is the causal neighborhood transition probability at the same scale; and $p\left(x_{s} \mid y_{d(s)}\right)$ is the partial posterior marginal probability.

For more details on proofs and conditional-independence assumptions, one could refer to the original formulation in Laferté et al. [7] for the quad-tree, or to the formulation in Hedhli et al. [16] for the multi-temporal case, or Hedhli et al. [15] for the MMRF case.

\section{EXPERIMENTAL RESULTS}

We applied the developed hierarchical classification approach to a multi-resolution dataset that consists of a panchromatic and a multi-spectral Pléiades images acquired over Port-au-Prince (Haiti). To fit with the dyadic decomposition imposed by the quad-tree, the finest resolution of the multi-resolution pyramid (level 0) was set equal to the finest resolution of the input panchromatic image (i.e. $0.5 \mathrm{~m}$ ). Co-registered multi-spectral images (at $2 \mathrm{~m}$ ) were integrated at level 2 of the pyramid. To employ the multi-resolution information, and to avoid an "empty" tree level (level 1), we integrate additional information by using a hierarchical decomposition (Daubechies 10 wavelet decomposition) of the panchromatic image.

Manually annotated non-overlapping training and test sets were selected by a remote sensing specialist in homogeneous areas (no borders were taken into account). Five classes were chosen: water, urban areas, vegetation, sand and containers. We compare the proposed method with the classification results obtained using the following techniques: 1) the original MPM formulation used by Laferté et al. [7]. In this case, the classification is obtained recursively over the scales and the use of a quad-tree in the MPM scheme yields blocky segmentations (details can be found in [11]), and 2) the contextual hierarchical approach using the unbalanced MMRF (details can be found in [15]).

The classification results are presented in Fig. 4 and numerically assessed via accuracy estimates (Tab. I). The classification map obtained when using the proposed approach shows satisfactory results, especially as compared to the original hierarchical classification technique based on the MPM criterion in [7] which yields blocky classification (see Fig. 4(b)). These artifacts are mitigated by incorporating spatial contextual information. The proposed method was also compared to an extended version of the method in [7] in which the blocky artifacts were reduced by employing an unbalanced MMRF [15]. Comparing to this method, the proposed technique mitigates the corner-dependent artifact due to the fact that each pixel depends on a non-symmetric neighborhood, as highlighted with black arrows in Fig. 4(c).

\section{CONCLUSION}

The proposed method is aimed at performing hierarchical classification using input multi-resolution imagery. It combines 


\begin{tabular}{|c|c|c|c|c|c|c|c|}
\hline & water \% & urban \% & vegetation \% & containers \% & soil \% & over all \% & computation time \\
\hline Proposed method & 100 & 92 & 89 & 81 & 94 & 91 & 147 seconds \\
\hline method in [7] & 100 & 62 & 76 & 72 & 91 & 80 & 120 seconds \\
\hline method in [15] & 100 & 74 & 83 & 86 & 92 & 87 & 154 seconds \\
\hline
\end{tabular}

TABLE I

RESUltS OBTAINED USING A PLÉIADES DATASET (IMAGE SIZES =1000 X 1600 PIXELS): CLASS ACCURACIES, OVERALL ACCURACY, AND COMPUTATION TIME.

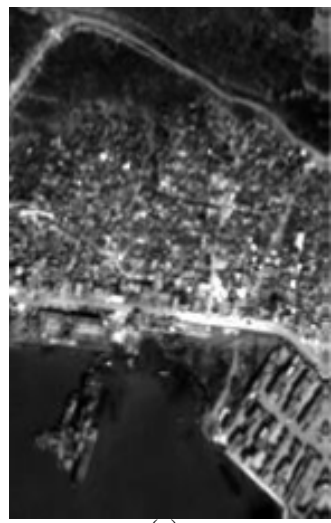

(a)

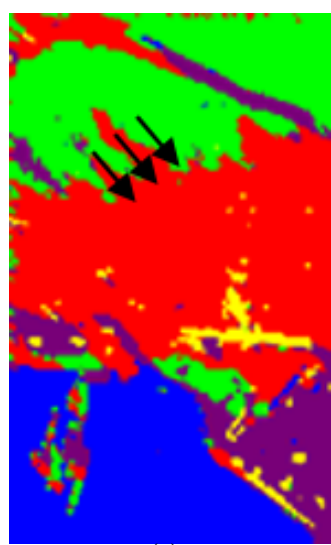

(c)

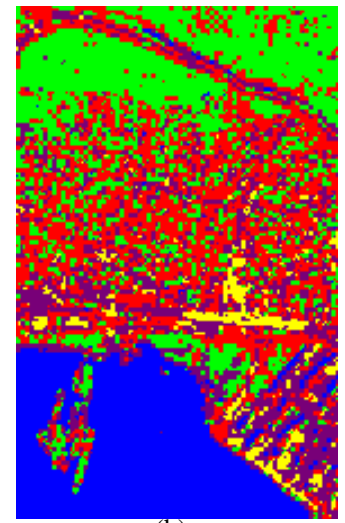

(b)

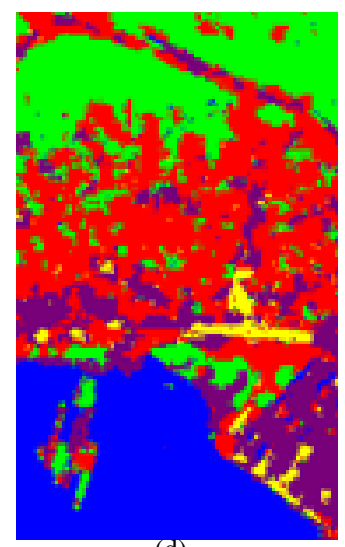

(d)
Fig. 4. classification maps of optical(Pléiades) image (a) using the original method proposed in [7] (b), the previous method in [15] (c) and the new proposed method (d).

a causal hierarchical MRF model using a quad-tree and a Symmetric Markov mesh to preserve contextual information at each scale by applying a non-iterative classification algorithm using the MPM criterion. Experimental results with HR satellite imagery of a very high-resolution urban scene suggest that the method allows to effectively incorporate spatial information in the hierarchical classification process and provides higher accuracies than previous techniques. Indeed, it is confirmed experimentally that MMRFs and their lattice models are corner-dependent, and that the proposed approach is effective in circumventing this drawback by using a Symmetric Markov Mesh Random Field. The proposed method, in the application to a challenging urban area classification problem, is demonstrated able to combine the computational and modeling benefits of hierarchical and symmetric mesh
MRF models, while preventing their individual artifacts.

\section{ACKNOWLEDGMENT}

The authors wish to thank the French Space Agency (Centre National des Etudes Spatiales, CNES) for providing the data used in the experiments and for partial financial support.

\section{REFERENCES}

[1] L. Gómez-Chova, D. Tuia, G. Moser, and G. Camps-Valls, "Multimodal classification of remote sensing images: a review and future directions," Proceedings of the IEEE, vol. 103, no. 9, pp. 1560-1584, 2015.

[2] C. Pohl and J. van Genderen, "Remote sensing image fusion: an update in the context of digital earth," International Journal of Digital Earth, vol. 7, no. 2, pp. 158-172, 2014.

[3] J. Clark and A. Yuille, Data fusion for sensory information processing systems. Springer Science \& Business Media, 2013, vol. 105.

[4] E. Waltz, J. Llinas et al., Multisensor data fusion. Artech house Norwood, MA, 1990, vol. 685.

[5] H.-C. Li, W. Hong, Y.-R. Wu, and P.-Z.-Fan, "On the empirical-statistical modeling of SAR images with generalized gamma distribution," IEEE J. Sel. Top. Signal Process., vol. 5, no. 3, pp. 386-397, 2011.

[6] M. Basseville, A. Benveniste, and A. Willsky, "Multiscale autoregresive processes, part i: Schur-levinson parametrizations, and part ii: Lattice structures for whitening and modelling," IEEE Transaction on Signal Processing, vol. 40, no. 8.

[7] J.-M. Laferté, P. Pérez, and F. Heitz, "Discrete Markov image modeling and inference on the quadtree," IEEE Transactions on Image Processing, vol. 9, no. 3, pp. 390-404, 2000.

[8] D. Pickard, "Unilateral markov fields," Advances in Applied Probability, pp. 655-671, 1980.

[9] Q. Razlighi, N. Kehtarnavaz, and A. Nosratinia, "Computation of image spatial entropy using quadrilateral Markov random field," IEEE Transactions on Image Processing, vol. 18, no. 12, pp. 2629-2639, 2009.

[10] S. Yousefi, N. Kehtarnavaz, and Y. Cao, "Computationally tractable stochastic image modeling based on symmetric markov mesh random fields," IEEE Transactions on Image Processing, vol. 22, no. 6, pp. 2192-2206, 2013

[11] P. Pérez, A. Chardin, and J.-M. Laferté, "Noniterative manipulation of discrete energy-based models for image analysis," Pattern Recognition, vol. 33, no. 4, pp. 573-586, 2000.

[12] K. Abend, T. J. Harley, and L. Kanal, "Classification of binary random patterns," IEEE Transactions on Information Theory, vol. 11, no. 4, pp. 538-544, 1965

[13] J. Besag, "Nearest-neighbour systems and the auto-logistic model for binary data," Journal of the Royal Statistical Society (B), pp. 75-83, 1972 .

[14] S. Yousefi and N. Kehtarnavaz, "Generating symmetric causal Markov random fields," Electronics letters, vol. 47, no. 22, p. 1, 2011.

[15] I. Hedhli, G. Moser, J. Zerubia, and S. Serpico, "Contextual multi-scale image classification on quadtree," in IEEE International Conference on Image Processing, 2016.

[16] I. Hedhli, G. Moser, J. Zerubia, and S. B. Serpico, "A New Cascade Model for the Hierarchical Joint Classification of Multitemporal and Multiresolution Remote Sensing Data ," IEEE Transactions on Geoscience and Remote Sensing, 2016, accepted for publication in the IEEE Transactions on Geoscience and Remote Sensing (TGRS). 